\title{
APLICACIÓN MÓVIL PARA LA RECOMENDACIÓN DE ASIGNATURAS EN LOS ESTUDIOS DE GRADO UNIVERSITARIOS
}

\section{Mobile APPlication FOR RECOMMENDING COURSES IN UNIVERSITY STUDIES}

\author{
A. Zafra, E. Gibaja, J.C. Gámez, \\ B. J.M. Soto, A. Arauzo, J.M. Moyano, \\ C. A. Ramírez, M. Martínez \\ azafra@uco.es \\ University of Cordoba
}

Received: 27/06/2017 Accepted: 20/02/2018

\begin{abstract}
Resumen
Los Grados Universitarios en sus planes de estudios tienen asignados una serie de créditos optativos, en los que el estudiante tiene libertad para elegir las asignaturas que más le interesen. Esta elección suele ser bastante complicada para los estudiantes, que si bien cuentan con una guía docente de cada asignatura, que les permite conocer los contenidos teóricos y prácticos, los profesores que la imparten, la metodología y los criterios de evaluación, no suele ser suficiente para decidirse. En este contexto, los estudiantes están interesados en las valoraciones que le pueden ofrecer otros compañeros o compañeras que las cursaron, siendo una práctica habitual que los estudiantes recurran a los estudiantes de otros cursos para que les orienten en el proceso. No obstante, se debe tener cierta precaución con esta práctica, porque no todas las valoraciones que se reciban deberían tener la misma consideración. Por un lado, los intereses y propósitos del estudiante que pide opinión y los del estudiante que la proporciona puede que sean diferentes, y por otro lado, puede que la información se encuentre distorsionada por haber pasado por varios intermediarios. En este escenario, los sistemas de recomendación aparecen como herramientas para ayudar a la toma de decisiones generando recomendaciones a los estudiantes sobre un determinado objeto de estudio, a partir de preferencias y opiniones dadas por otros estudiantes que tienen unos intereses o preferencias similares a los suyos.

En este proyecto se ha diseñado e implementado una aplicación móvil, fácil de manejar por los estudiantes y que permite que cualquier estudiante registrado en un determinado Grado pueda obtener una recomendación sobre las asignaturas que más le pueden interesar.

Palabras clave: Recomendador colaborativo; Educación; Estudios universitários.

Abstract

University studies contain several optional subjects that can be chosen by students according to their preferences. To make this decision, student has a teaching guide of each subject which contains information about theoretical and practical contents, professors, methodology and assessment process. Despite being useful, in many cases this information is insufficient to make the best decision. In this context, students ask other peer students who have already studied the subject for advice. However, this process should be carried out cautiously. On one hand, the interests and goals of consulted students could be different to those of the student that requires the information, and on the other hand, the information could present a distorted vision of reality due to the intervention of many intermediaries.

This project intends to offer recommendations that help students to choose the subjects that could be more interesting for him or her from opinions given by other peers. With this purpose, we design and develop a mobile application that allows students to obtain a recommendation about the more convenient subjects according to his/her preferences.
\end{abstract}

Keywords: Collaborative recommender; Education; University study.

\section{INTRODUCCIÓN}

En la era tecnológica en la que vivimos, donde una gran cantidad de información es almacenada diariamente en todos los ámbitos de nuestra vida, nos encontramos con una sobrecarga de información, la cual no somos capaces de procesar. Esta información que, a priori, puede resultar valiosa para la toma de decisiones es muy amplia y está dispersa. Por este motivo, analizarla para obtener conocimiento real se convierte en una tarea complicada.

Los sistemas de recomendación [1], aparecen como herramientas que nos ayudan a buscar productos o servicios que puedan ser de nuestro interés y que tienen en cuenta nuestras preferencias particulares. Existen diversos tipos de sistemas de recomendación, los cuales difieren en el método o proceso de obtención de las recomendaciones y/o en las fuentes de información usadas, pudiendo destacar: sistemas de recomendación colaborativos [2], sistemas basados en contenido [3], en información demográfica [4], en conocimiento [5], o hibridando alguna de estas técnicas [6]. Los sistemas de recomendación colaborativos, que son en los que nos centraremos en este proyecto, son ampliamente utilizados debido a su simplicidad y sus buenos resultados. Dichos sistemas sugieren nuevos productos o servicios para un usuario particular, basándose en sus elecciones anteriores y en las elecciones que otros usuarios con un similar historial de valoraciones han realizado. De este modo, se estima la valoración que un usuario daría a un producto, en base al valor que asignaron sobre dicho producto una serie de usuarios con unos intereses o preferencias similares.

En este contexto, y con toda la información que tenemos a nuestra disposición, las aplicaciones de recomendación se han convertido en herramientas necesarias que se pueden encontrar en una gran variedad de ámbitos, sobre todo en el comercio electrónico y el ocio. Podemos encontrar ejemplos en la recomendación de libros, películas, noticias, música y viajes [7, 8]. 
En este proyecto, nos centraremos en el diseño de sistemas recomendación aplicados al ámbito educativo. Aunque la idea es extensible a cualquier nivel educativo, ya sea bachillerato, ciclo o máster, el trabajo se centra en los estudios de Grado Universitarios. En cualquiera de los Grados Universitarios que se encuentran disponibles, existen una serie de asignaturas que todos los estudiantes están obligados a cursar. Junto a estas asignaturas obligatorias, encontramos otras conocidas como optativas, entre las que los estudiantes pueden elegir aquellas que les resulten más interesantes. Esta decisión normalmente resulta complicada porque no cuentan con toda la información que les gustaría tener de una forma organizada. Por un lado, tienen a su disposición una guía docente de cada una de las asignaturas, donde pueden encontrar los contenidos teóricos y prácticos, el profesorado, la metodología y los criterios de evaluación. Por otro lado, cuentan con otra información que tiene más incertidumbre, y son las recomendaciones que reciben de compañeros o compañeras que ya las han cursado. Tenemos que tener presente, que muchas veces, las recomendaciones que llegan por el boca a boca pueden estar desvirtuadas, debido a que cada intermediario añade su propia impresión. Impresión, que además, puede estar supeditada a sus propios intereses, los cuales pueden ser muy diferentes de los de la persona que recibe la valoración. En definitiva, no se trata de una tarea trivial, una asignatura puede ser más o menos atractiva, en función de su contenido, de cómo se imparta, de la metodología de trabajo, de los criterios de evaluación, de quién la imparta y por supuesto, de los objetivos que el alumno pretende conseguir al cursarla (aprender, aprobar, obtener una calificación alta, ...). Son muchos los factores que pueden hacer que una asignatura sea atractiva para unos alumnos, pero no lo sea tanto para otros.

Es en este punto, donde un sistema de recomendación, que aprenda los intereses de un estudiante, encuentre valoraciones de estudiantes que tienen unos intereses en común y realice una recomendación de las asignaturas en función de dichas valoraciones, podría resultar una herramienta de gran utilidad. Con la idea de conseguir esta finalidad, este proyecto ha realizado el diseño e implementación de una aplicación móvil que recomienda asignaturas a los estudiantes. Para que la aplicación sea flexible y práctica, se ha diseñado una parte de administrador que permite introducir, eliminar o modificar los estudios de Grado que se contemplan, las asignaturas, los profesores y los departamentos, configurar los sistemas de recomendación que se utilizarán y obtener informes de salida que permiten obtener información representativa de los resultados almacenados. Desde el punto de vista del estudiante, se permite introducir valoraciones de las asignaturas cursadas, ver el ranking de las asignaturas, y obtener recomendaciones de las asignaturas que mejor se adapten a sus preferencias.

\section{OBjeTIVOS}

La principal meta que se pretende alcanzar con este proyecto es ayudar al estudiante a decidir las asignaturas optativas que le sería más conveniente cursar. Con esta finalidad, se plantea como objetivo el diseño e implementación de una aplicación móvil para la recomendación de asignaturas optativas basándonos en un sistema de recomendación colaborativo. De este modo, el sistema recomendará las asignaturas, en función de las valoraciones que una serie de alumnos con intereses similares han realizado de las mismas.

Para poder obtener unos resultados preliminares, se diseñará una encuesta donde los alumnos de tercer y cuarto curso del Grado de Ingeniería Informática podrán valorar las asignaturas que hayan cursado y esa información inicial será introducida en la base de datos, como información inicial que será empleada por el sistema de recomendación.

A continuación, se detallan las opciones que consideramos que debe incluir la aplicación para cada uno de los usuarios de la misma. Para los usuarios con el rol de administrador, la herramienta debe permitir:

- Introducir, eliminar o modificar estudios de Grado. Para cada uno de los estudios de Grado considerados, se podrá añadir, eliminar o modificar las asignaturas que lo componen.

- Modificar el algoritmo de recomendación que se utilice para realizar las recomendaciones, permitiendo modificar la configuración del mismo.

- Hacer copias de seguridad de la base de datos, para que toda la información que se tiene almacenada se mantenga de forma segura.

- Obtener informes de salida, que nos indique para cada Grado las asignaturas que han sido más valoradas, o en las que se han obtenido mejores calificaciones medias por los estudiantes. Esta información puede ser interesante también para otros servicios con los que cuenta la Universidad, como podría ser coordinadores de titulación o unidades de garantía de calidad, por citar algunos.

Para los usuarios con el rol de estudiante, la herramienta debe permitir:

- Registrarse como usuario, introduciendo su información personal y el Grado en el que se encuentra matriculado.

- Introducir para las diferentes asignaturas que ha cursado, tanto básicas, como obligatorias, como optativas, la calificación que obtuvo, el profesor que la impartió y la valoración que le asigna.

- Proporcionar recomendaciones de las asignaturas que más le puedan interesar.

- Obtener el ranking de las asignaturas mejor valoradas. 
Otros requisitos que se deben tener en cuenta para el diseño e implementación de la aplicación son:

- La aplicación será diseñada para dispositivos móviles que dispongan del sistema operativo Android.

- El diseño de la aplicación será modular, de modo que permita ser fácilmente modificable y ampliable. Debe permitir la escalabilidad de modo que pueda albergar nuevos módulos que puedan resultar de utilidad.

- La interfaz, además de ser sencilla e intuitiva, de manera que pueda ser gestionada sin que se requieran conocimientos técnicos avanzados tanto para la administración, como para el resto de usuarios, mantendrá una apariencia similar a aplicaciones que los estudiantes están acostumbrados a utilizar.

\section{METODOLOGÍA}

Para alcanzar los objetivos previamente comentados, se han realizado las siguientes actividades:

- Se ha diseñado y desarrollado una base de datos. Esta base de datos almacena toda la información referente a los Grados, las asignaturas, los profesores, los departamentos, los usuarios y sus preferencias y calificaciones.

- Se ha desarrollado una aplicación móvil accesible por los administradores que permite su configuración: introducir, eliminar o modificar información de los diferentes Grados, asignaturas, profesorado, departamentos, configuraciones del sistema de recomendación, así como obtener informes de salida.

- Se ha desarrollado una aplicación móvil accesible por los estudiantes para poder recibir las recomendaciones acerca de las asignaturas que les pueden resultar más interesantes. El acceso a esta aplicación requiere el registro del estudiante en un determinado Grado y la introducción inicial de las valoraciones de las asignaturas que ya haya cursado. Normalmente, las asignaturas optativas no son seleccionadas en los primeros cursos, con lo que cuando el estudiante necesita tomar esta decisión ya ha cursado y ha podido valorar una serie de asignaturas de los primeros cursos, que permite que el sistema tenga información de sus intereses. Además, se permite una actualización de la información de cada alumno en un proceso de retro-alimentación.

- Se ha estudiado las librerías de algoritmos de recomendación más relevantes, y se ha seleccionado finalmente la librería Mahout [9].

- Se han elaborado y realizado encuestas. Debido a que los sistemas de recomendación colaborativos necesitan tener valoraciones iniciales para poder realizar una recomendación, se han recogido mediante encuestas en papel, las valoraciones de los estudiantes de tercer y cuarto curso del Grado de Ingeniería Informática. La información se ha almacenado en la base de datos y ha servido para poder llevar a cabo un estudio experimental preliminar.

- Se ha validado el sistema. Con los datos recogidos se han analizado los resultados obtenidos y se han evaluado diferentes configuraciones del algoritmo de recomendación empleado.

\section{DESCRIPCIÓN}

La aplicación desarrollada, WhatSubject, se ha desarrollado para sistemas Android y hace uso de la librería Mahout[9], y cumple con todos los objetivos planteados. Existen dos tipos distintos de usuarios en nuestro sistema: el usuario administrador y el usuario alumno. A continuación, se van a describir las principales funcionalidades a las que pueden acceder cada uno de estos usuarios.

\subsection{USUARIO ADMINISTRADOR}

El usuario administrador puede introducir nuevos grados que se consideren en la aplicación. Para cada grado, se permite tanto la introducción, como la modificación, como la eliminación de la información relativa a las asignaturas, profesores y departamentos implicados. En la Figura 1, se muestra como acceden estos usuarios al sistema. Una vez que la conexión del administrador ha sido validada con su usuario y contraseña, nos aparecerá la pantalla principal del administrador (Figura 2). A continuación, se detallarán las diferentes opciones implementadas para el administrador.

- Acceso al panel de administración (Dashboard). En la pantalla dashboard, se puede llevar a cabo determinadas configuraciones básicas en el algoritmo de recomendación que se emplea, como son: seleccionar la métrica de distancia a utilizar y el número de vecinos. También, se pemite ver el ranking de valoraciones o de notas medias de las asignaturas, realizar una copia de seguridad de la base de datos y descargar dicha copia. 


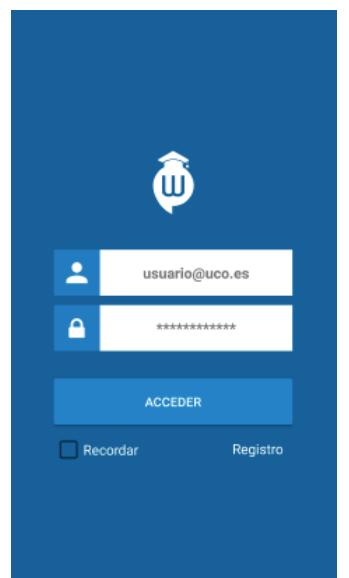

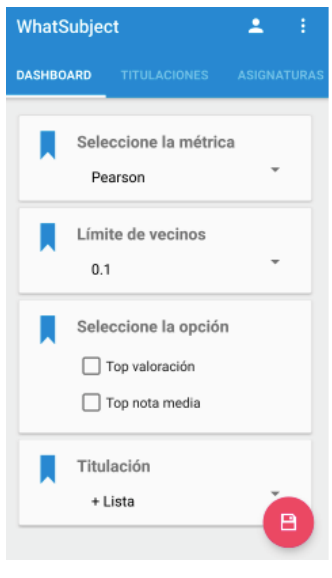

Figura 2. Panel de administración

Figura 1. Iniciar sesión

- Acceso al panel de titulaciones. En la pantalla titulaciones se puede añadir, editar o eliminar una titulación. La opción de añadir, permite introducir un nuevo Grado especificando el nombre del mismo. En las opciones de editar y eliminar, se debe seleccionar uno de los Grados ya establecidos, y realizar la modificación o eliminación del mismo.

- Acceso al panel de asignaturas. En la pantalla asignaturas se puede añadir, editar o eliminar una asignatura. La opción de añadir, te permite introducir una nueva asignatura, especificando su nombre, curso, titulación y el departamento al que pertenece. En el caso de editar, se debe seleccionar una asignatura dentro de las disponibles, y modificar cualquiera de sus campos. Finalmente, eliminar, nos permite seleccionar una asignatura de una determinada titulación, y eliminarla.

- Acceso al panel de departamentos. En la pantalla departamentos se puede añadir, editar o eliminar un departamento. La información de un departamento hace referencia a su nombre, el cual podrá ser editado o eliminado, una vez que se haya añadido.

- Acceso al panel de profesores. En la pantalla profesores se puede añadir, editar o eliminar un profesor. La información requerida para añadir un nuevo profesor, será su nombre, apellidos, y el departamento al que pertenece. Esta información podrá ser modificada o eliminada en todo momento, seleccionando la opción oportuna.

\subsection{Usuario Alumno}

Los alumnos ya registrados acceden a la aplicación a través de su usuario y contraseña (Figura 1). El acceso es idéntico al de los administradores, con la salvedad de que los usuarios alumnos pueden registrarse ellos directamente, en caso de no estar registrados en el sistema, utilizando la opción de registro de la ventana principal (Figura 1). En el proceso de registro (Figura 3), deben indicar la titulación en la que están matriculados, su correo electrónico, su nombre y sus apellidos.

Una vez validado el alumno, se le muestra diferente funcionalidad que puede llevar a cabo (Figura 4), y que se detalla a continuación:

- Acceso al panel de asignaturas puntuadas. En la pantalla de asignaturas puntuadas, se muestran las asignaturas que previamente han sido valoradas por el usuario. En caso de no haber valorado todavía ninguna asignatura, aparecerá un aviso indicándolo.

- Acceso al panel de asignaturas pendientes. En la pantalla de asignaturas pendientes de valorar, se muestran las asignaturas que todavía no han sido valoradas por el usuario. Cada vez que se valore una asignatura localizada en pendientes, se eliminará de esta sección, y se incluirá en la sección de puntuadas.

- Acceso al panel de asignaturas recomendadas. En la pantalla de asignaturas recomendadas, se muestran, en función de las preferencias de cada estudiante, las asignaturas que se le recomiendan. En caso de no existir suficiente información para realizar la recomendación, debido a que no se dispone de suficientes valoraciones de dicho estudiante, aparecerá un mensaje indicándolo. 
- Valorar asignatura. Si se desea valorar una nueva asignatura, se debe seleccionar una asignatura en la pestaña de pendientes de

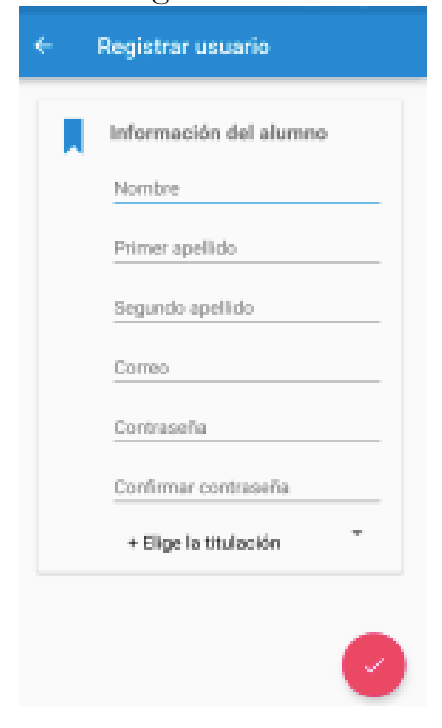

Figure 3. Panel de registro de alumnos

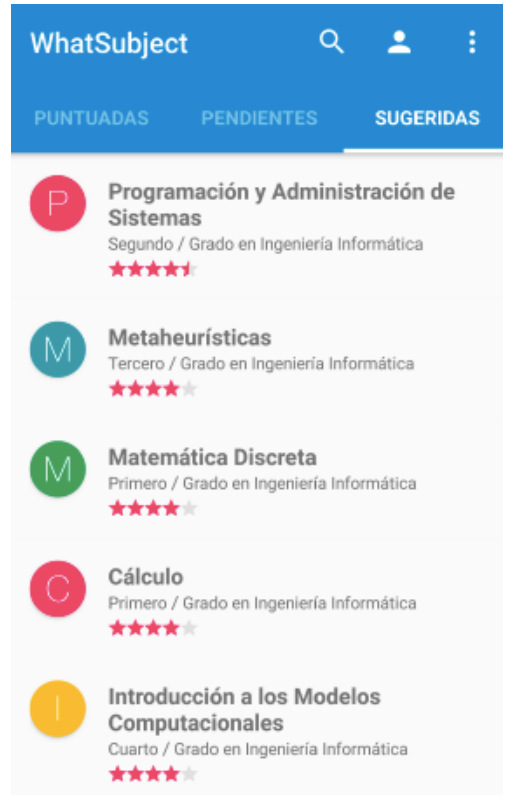

Figura 5. Panel de usuario (asignaturas recomendadas)

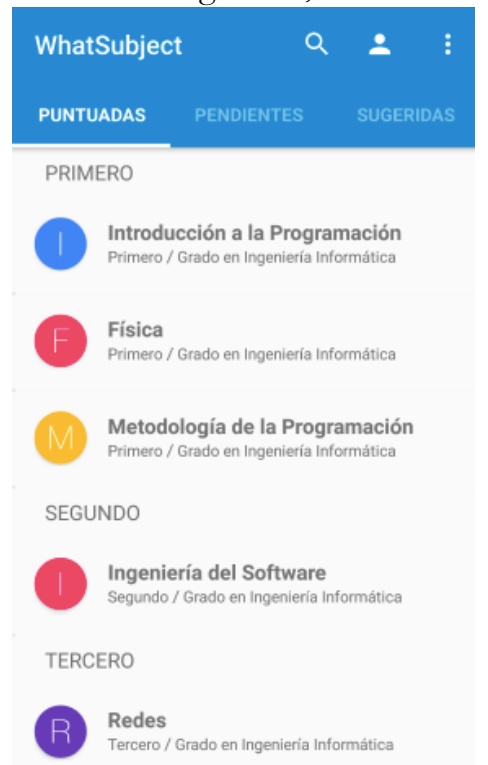

Figura 4. Panel de usuario

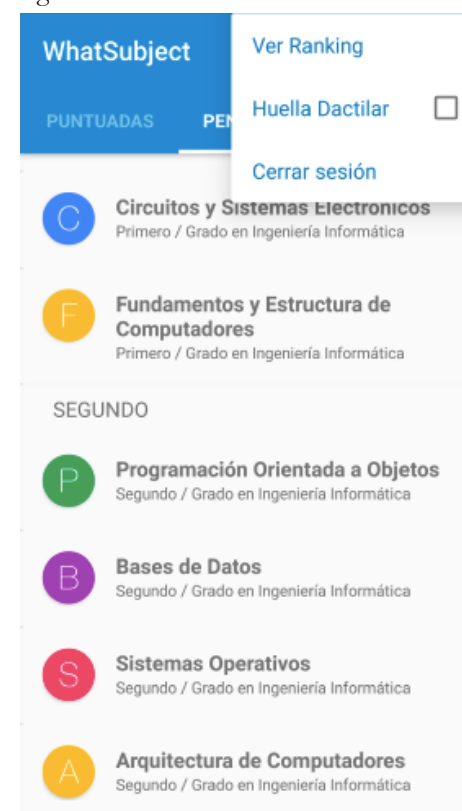

Figura 6. Panel de usuario. Menú principal valorar. Si se desea modificar una valoración realizada asignatura de la pestaña de ya valoradas. Al seleccionar la asignatura, se accede a una pantalla que te permite indicar la valoración, el profesor o profesores que la han impartido, y la calificación obtenida (Figura 6). Una vez incluida la información, se debe confirmar la operación.

- Buscar asignatura. Para buscar una asignatura, se debe de seleccionar la lupa, donde se puede introducir un texto a buscar. Esta funcionalidad muestra todas las asignaturas que contienen en su nombre el texto introducido.

- $\quad$ Opciones de menú. En la parte superior de la pantalla se muestra un menú desplegable en el cual podemos seleccionar una serie de opciones, entre ellas ver el ranking de las asignaturas, activar la huella dactilar o cerrar sesión (Figura 7).

- Ver el ranking de asignaturas. El usuario alumno también podrá ver un ranking de asignaturas, bien por valoraciones obtenidas, bien por calificaciones obtenidas por los usuarios.

- Activar huella dactilar. Si se pulsa sobre la opción del menú Huella Dactilar, se puede vincular una huella dactilar con la aplicación. Una vez vinculada la huella dactilar con la aplicación, al iniciar sesión se permitirá la opción de iniciar mediante la huella dactilar sin necesidad de introducir las credenciales.

- Ver perfil. Si se pulsa sobre la figura de la imagen en la parte superior de la pantalla, se accede al perfil del usuario. La información del perfil se puede editar pulsando el icono del lapiz. En la pantalla de editar perfil, se puede modificar los campos del perfil de usuario, que coincide con los introducidos en el registro.

\section{RESULTADOS OBTENIDOS}

Durante este curso académico, y para partir de una información inicial, necesaria en la recomendación colaborativo, se ha recogido y almacenado información del Grado implicado y de las valoraciones de los estudiantes, para poder obtener unos primeros resultados preliminares, que nos permitan evaluar el algoritmo de recomendación utilizado con diferentes configuraciones y métricas. Se debe clarificar, que los algoritmos de recomendación, funcionan mejor, cuanta más información tienen almacenada. Por tanto, se espera que conforme más valoraciones sean introducidas, mejores podrán ser los resultados obtenidos.

\subsection{Datos UTILIZADOS}

El estudio experimental se ha realizado en el Grado en Ingeniería Informática, considerando las asignaturas de sus tres especialidades (Computación, Computadores e Ingeniería de Software). De acuerdo, a estos estudios universitarios: existen una serie de asignaturas comunes a las tres especialidades, concretamente 25 asignaturas, cada especialidad cuenta con 8 asignaturas específicas, y finalmente existen 14 asignaturas optativas, de las que deben seleccionar solamente 6. En este estudio experimental, participaron 28 alumnos entre tercer y cuarto curso, de las diferentes especialidades. Estos alumnos realizaron una encuesta donde pusieron las valoraciones de las asignaturas que ya habían cursado, junto con las calificaciones obtenidas y los profesores que las impartieron. Esta información fue incorporada a la base de datos utilizada en la aplicación, simulando así el comportamiento si hubiese estado operativa durante este curso académico, y los alumnos hubiesen introducido su información. 
Un resumen de la información recogida, se muestra en la Tabla 1. Esta tabla muestra también el porcentaje de asignaturas valoradas por cada alumno. Para calcular estos porcentajes, para cada alumno, solamente se ha considerado las asignaturas potenciales que podría valorar, de acuerdo a su especialidad, y al número máximo de optativas que puede cursar.

\section{Grado de Ingeniería Informática}

\begin{tabular}{|c|c|c|}
\hline \multirow[t]{3}{*}{ Asignaturas } & Optativas & 14 (6 se deben seleccionar) \\
\hline & Comunes & 25 \\
\hline & Especialidad & 24 (8 por especialidad) \\
\hline \multirow{3}{*}{$\begin{array}{ll}\text { Porcentaje de las } \\
\text { asignaturas valoradas }\end{array}$} & Media & $84.38 \%$ \\
\hline & Máximo & $100 \%$ \\
\hline & Mínimo & $53.12 \%$ \\
\hline
\end{tabular}

Tabla 1. Resumen de los datos utilizados

\subsection{CONFIGURACIÓN DEL ALGORITMO}

Para llevar a cabo este estudio experimental, se van a considerar diferentes configuraciones del algoritmo desarrollado. Concretamente, se han evaluado diferentes métricas de similaridad y diferentes umbrales que determinan el número de vecinos. Concretamente, las métricas utilizadas son [9]:

- Correlación de Pearson. Mide el grado de relación entre dos variables, determinando si existe una relación positiva, negativa, o no existe ninguna relación entre los usuarios.

- Distancia Euclidea. Nos permite determinar cómo las valoraciones de un usuario están alejadas o cerca de las valoraciones de otro, para determinar cuáles son los usuarios con valoraciones más próximas.

- Coeficiente de Tanimoto. Nos permite determinar si existe similaridad porque los usuarios han preferido los mismos ítems, o si no existe porque no tienen ítems preferidos en común.

- Probabilidad LogLikelihood. Nos permite determinar el número de ítems que dos usuarios prefieren, el número de ítems que el segundo usuario prefirió y el primero no, el número de ítems que el primer usuario prefirió y el segundo no, y el número de ítems que ninguno de los usuarios prefirió.

Con respecto a la selección de vecinos, Mahout [9], nos permite seleccionar directamente el número de vecinos que se quiere emplear o determinar un umbral para la sección de vecinos. En nuestro estudio, se trabajará con el umbral, y para evaluar su efecto en la realización de la recomendación se emplearán varios valores. Concretamente, los valores utilizados han sido 0.1, 0.5 y 0.9. Como en Mahout las medidas de similaridad entre dos usuarios viene dada entre -1 y 1 , estos umbrales nos permiten seleccionar sólo los vecinos que lleguen a un determinado nivel de similaridad. Cuanto más próximo a 1 es el umbral, será necesario que los vecinos sean más próximos al usuario del que se va a realizar la recomendación, con lo que un menor número de vecinos suele ser seleccionado.

\subsection{MÉTRICAS CONSIDERADAS}

Las métricas utilizadas para evaluar los resultados de las recomendaciones realizadas, son [9]:

- Error Medio Absoluto (Mean Absolute Error, MAE), estima la exactitud con la que el sistema realizará las recomendaciones.

- Precisión, es la proporción de las recomendaciones que se le han proporcionado al alumno y las que realmente eran de su interés.

- Recall, es la proporción de entre todos los elementos de interés del alumno, y las recomendaciones que le ha realizado el sistema.

\subsection{ANÁLISIS DE LOS RESULTADOS}

Para comprobar la validez de las recomendaciones, se ha llevado a cabo un proceso de validación, donde un $90 \%$ de los datos es utilizado para llevar a cabo el entrenamiento del modelo, y el 10\% restante es obtenido para determinar los resultados de las recomendaciones realizadas.

La Tabla 2 muestra los resultados obtenidos considerando las 4 métricas de similaridad y los diferentes umbrales para determinar el número de vecinos que se han evaluado. Los valores de MAE calculan la diferencia real entre el valor de interés indicado por el usuario (de 1 a 5) y el estimado por los algoritmos de recomendación. En el caso de la Precisión y Recall, para controlar los aciertos y los fallos, se considera un umbral que establece como relevante, aquella asignatura que tenga una valoración mayor que las valoraciones medias de dicho usuario.

\begin{tabular}{|c|c|c|c|c|}
\hline Similaridad & Umbral & MAE & Precisión & Recall \\
\hline Pearson & 0.1 & 0.9262 & 0.6047 & 0.7190 \\
\hline Euclidea & 0.1 & 0.9154 & 0.4761 & 0.6404 \\
\hline
\end{tabular}




\begin{tabular}{|l|c|c|c|c|}
\hline Tanimoto & 0.1 & 0.9294 & 0.4761 & 0.6404 \\
\hline logLikelihood & 0.1 & 0.9323 & 0.4920 & 0.6523 \\
\hline Pearson & 0.5 & 0.9003 & 0.8666 & 0.6809 \\
\hline Euclidea & 0.5 & 0.8007 & 0.7904 & 0.5174 \\
\hline Tanimoto & 0.5 & 0.9300 & 0.6425 & 0.7269 \\
\cline { 2 - 5 } logLikelihood & 0.5 & 0.9330 & 0.5833 & 0.7023 \\
\hline Pearson & 0.9 & - & - & 0.0000 \\
\hline Euclidea & 0.9 & - & - & 0.0000 \\
\hline Tanimoto & 0.9 & 0.5000 & 0.7916 & 0.1904 \\
\hline logLikelihood & 0.9 & 0.9365 & 0.6789 & 0.7214 \\
\hline
\end{tabular}

Tabla 2. Resultados experimentales (similaridad/umbral)

Se puede ver que los resultados con un umbral de 0.9 muchas veces no pueden ser calculados, debido a que el umbral es muy próximo a 1.0 (valor de similaridad máximo), y por tanto, los vecinos que se consideran deben tener ese nivel de similaridad para poder ser considerados. Al tener que ser tan similares, y contar con tan pocos usuarios iniciales, a veces no se encuentra el número suficiente, y por tanto los cálculos no pueden ser realizados. Si evaluamos las métricas, se puede apreciar que los mejores valores son obtenidos cuando se trabaja con la similitud del coeficiente de Pearson. Con respecto a los umbrales, los mejores resultados se consiguen con un umbral de un 0.5. La combinación de Pearson con un umbral de 0.5 , ha obtenido un $86.6 \%$ de precisión, lo que indica que de lo que se le recomendó al estudiante, el $86.6 \%$ realmente era de su interés y una precisión del $68.1 \%$, lo que indica que de todo lo que le realmente le interesa al estudiante, se le recomendó casi el 70\%. Estos resultados, resultan prometedores, considerando la poca información a priori que se posee, ya que el número de estudiantes empleado en el estudio es muy reducido, considerando además que son de diferentes especialidades, y en cada especialidad las asignaturas cursadas son diferentes. Con estas premisas, se considera que los resultados son muy positivos, y que sería interesante contar con más información, para poder hacer un estudio más preciso, y conseguir mejorar los resultados.

\section{CONCLUSIONES}

La aplicación desarrollada realiza recomendaciones a los estudiantes de los Grados Universitarios de las asignaturas optativas que más se adaptan a sus intereses. Para llevar a cabo la recomendación, se consideran las valoraciones que otros estudiantes que ya han cursado esas asignaturas le han asignado. Además, se ha incluido en la aplicación desarrollada funcionalidad, tanto a nivel de administrador, como a nivel de estudiante, que hacen que la aplicación sea flexible para adaptarse a diferentes estudios o a modificaciones que vayan sucediendo.

Para evaluar la aplicación, se ha llevado a cabo un estudio experimental en el Grado en Ingeniería Informática de la Universidad de Córdoba, obteniendo unos resultados prometedores. En el estudio preliminar que se ha realizado, se han comparado diferentes configuraciones del algoritmo de recomendación, y se han evaluado diferentes métricas, mostrando que la aplicación podría ser de gran utilidad para los estudiantes.

\section{AGRADECIMIENTOS}

Proyecto de innovación de la Universidad del Córdoba del curso académico 2016/2017.

\section{BIBLIOGRAFÍA}

[1] F. RICCI, L. ROKACH, B. SHAPIRA, P.B. KANTOR. Recommender systems handbook, Springer, ISBN: 978-0-387-85819-9, 2011.

[2] B. XU, J. BU, C. CHEN, D. CAI. An exploration of improving collaborative recommender systems via user-item subgroups. In Proceedings of the 21st international conference on World Wide Web, ACM, 2012, p. 21-30).

[3] P. Lops, M. De Gemmis, G. Semeraro. Content-based recommender systems: State of the art and trends. In Recommender systems handbook, 2011, Springer, p. 73-105.

[4] J.B. SChAfer, J. A. Konstan, M.J. PAZZAni. A framework for collaborative, content-based and demographic filtering. Artificial Intelligence Review 13 (5-6), 1999, p.393-408.

[5] R. BURKE, Knowledge-based Recommender Systems. Encyclopedia of Library and Information Systems, 2000.

[6] M. BArranco, L. G. PÉreZ, L. MartínEZ. Un Sistema de Recomendación Basado en Conocimiento con Información Lingü.stica Multiganular. Paper read at SIGEF XIII - The XIII Congress of International Association for Fuz:y-Set Management and Economy, at Hammamet, Tunicia, 2006. [7] J. LiU, P. DOLAN, E.R. PEDERSEN. Personalized news recommendation based on click behavior. In Proceedings of the 15th international conference on Intelligent user interfaces, 2010, p. 31-40.

[8] A. MAJID, L. ChEn, G. CHEN, H.T. MiRZA, I. Hussain, J. WOODWARD. A context-aware personalized travel recommendation system based on geotagged social media data mining. International Journal of Geographical Information Science, 27(4), 2013 , p. 662-684.

[9] S. Owen, R. ANIL, T. DunNING. Mahout in Action, ISBN 978-1-935-18268-9, Manning Publications co., 2012. 